\title{
Evolución de los departamentos de comunicación en las editoriales
}

\author{
Por Juan-Carlos Marcos-Recio, Juan-Miguel Sánchez-Vigil y María Olivera-Zaldua
}

\begin{abstract}
Resumen: Se presenta la evolución de los departamentos de comunicación en las editoriales durante la última década, después de que el libro electrónico irrumpiera en el sector. Se exponen las tareas que se realizan habitualmente y la problemática derivada de la actividad. Para el análisis se han recogido y analizado las opiniones de los directores y jefes de prensa de Comunicación de Santillana (Rosa Junquera-Santiago), Random House Mondadori (Leticia Rodero) y Literal Press (Lola Martín).
\end{abstract}

Palabras clave: Comunicación, Comunicación editorial, Edición, Publicidad editorial, Marketing editorial.

Title: Evolution of communication departments in publishing companies

Abstract: The purpose of this article is to determine the evolution of Communications Departments in publishing companies over the past decade, after the appearance of the e-book in the sector. The work customarily carried out is covered, along with the everyday problems that arise. We have obtained and analyzed the opinions of the following Managers and Press Secretaries for this study: Santillana Communications (Rosa

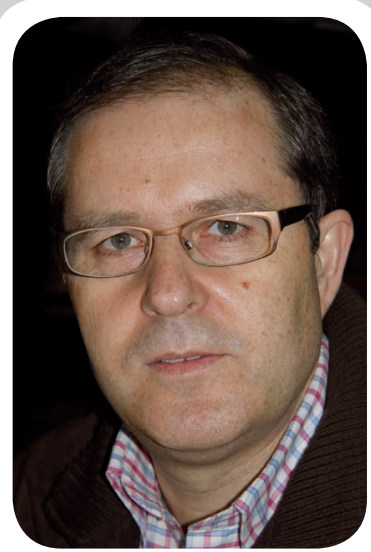

Juan-Carlos Marcos-Recio es profesor contratado doctor del Depto. de Biblioteconomía y Documentación de la Univ. Complutense de Madrid. Es especialista en documentación en medios, documentación digital en medios y experto en educación a distancia. Su línea de investigación actual es la documentación y la investigación en la publicidad, tema sobre el que ha publicado varios libros: La documentación electrónica en los medios de comunicación (1999); Gestión de la documentación en la publicidad y en las relaciones públicas (2004), y artículos: "Evolución y desarrollo de la documentación en el campo publicitario: recursos para optimizar una campaña" (2002) y "Estrategias documentales en agencias de publicidad" (2003). Es creador y director de Publidocnet (Centro Documental para la Conservación del Patrimonio Publicitario Español)

http://www.publidocnet.com

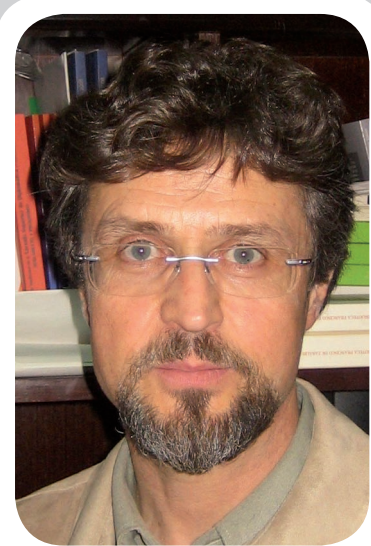

Juan-Miguel Sánchez-Vigil es doctor en ciencias de la información por la Facultad de Ciencias de la Información de la Univ. Complutense. Es profesor contratado doctor del Departamento de Biblioteconomía y Documentación de la misma universidad. Editor y fotógrafo, colaborador de la editorial Espasa-Calpe y de varias revistas especializadas, entre ellas la literaria Ínsula. En relación con la edición ha publicado La edición en España (2009) y Calpe, paradigma editorial (2005), y ha coordinado el monográfico de la revista Ínsula dedicado a la Colección Austral (2009). Sobre documentación fotográfica e iconográfica ha publicado Revistas ilustradas en España (2008), Del daguerrotipo a la Instamatic (2007), El documento fotográfico (2006) y Diccionario de fotografía (2002). Es coordinador junto a Juan-Carlos Marcos-Recio del blog Documentación. http://weblogs.madrimasd. org/documentacion

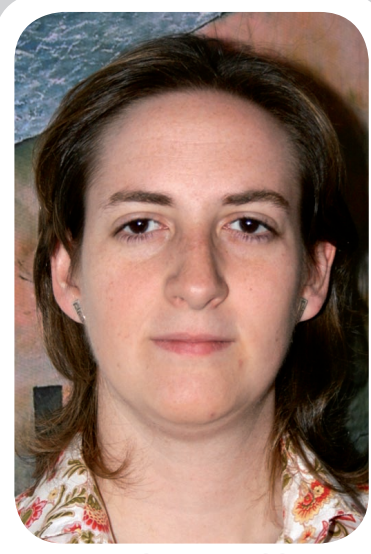

María Olivera-Zaldua es licenciada en documentación por la Facultad de Ciencias de la Documentación de la Universidad Complutense. Estudiante de Doctorado en la misma facultad con el DEA Archivo iconográfico de Joaquín Turina. Documentalista de la Editorial Espasa-Calpe en las obras Los Toros de Cossío y Los Episodios Nacionales de Pérez-Galdós. Forma parte del equipo de investigadores del Archivo del Ateneo de Madrid. Ha colaborado en los libros Revistas ilustradas en España y Del daguerrotipo a la Instamatic, y es coautora del artículo "Compromiso social de las bibliotecas frente a la industria cultural: biblioteca Fundación Juan March" (Fesabid, 2009). En las III Jornadas sobre Ateneístas llustres (2008) pronunció la conferencia: Nicolás María Urgoiti. Es colaboradora del blog Documentación.

http://weblogs.madrimasd. org/documentacion Junquera Santiago), Random House Mondadori (Leticia Rodero) and Literal Press (Lola Martín).

Keywords: Communications, Editorial communications, Publishing, Editorial advertising, Editorial marketing.

Marcos-Recio, Juan-Carlos; Sánchez-Vigil, Juan-Miguel; Olivera-Zaldua, María. “Evolución de los departamentos de comunicación en las editoriales”. El profesional de la información, 2010, enero-febrero, v. 19, n. 1, pp. $52-56$.

DOI: 10.3145/epi.2010.ene.07

\section{Introducción}

LOS AVANCES EN LA CIVILIZACIÓN han tenido como referentes diversos materiales tangibles en los siglos pasados (carbón, acero, petróleo) e intangibles en la actualidad, siendo la información la materia primera que desde la llegada de internet revoluciona la sociedad.
¿Qué tienen en común las empresas, las instituciones, las universidades, las organizaciones no gubernamentales (ONGs) y hasta el propio Gobierno? ¿Qué es lo 
primero que valoran? y ¿cómo se enfrentan a ella en un mundo en el que la abundancia de información está abrumando cada vez más la sociedad? Estos organismos tienen resuelta una parte importante de su gestión si cuentan con un gabinete de comunicación que sea capaz de tratar la información. La COMUNICACIÓN, en letras mayúsculas, la comunicación global, con criterio y con un valor de marca, es el eje sobre el que se vertebra un camino hacia el consumidor, el usuario, la persona y la sociedad.

Pero comunicar no es fácil, o no lo es hacerlo en los valores de una marca, mediante la motivación, con respeto a las tareas sociales y con criterios éticos. Aunque se pretendan crear modelos únicos, cada acto de comunicación requiere actuaciones diferentes. En todo caso, lo importante de la comunicación está en dos frentes: hacia fuera, cuyo esfuerzo ha de ser calculado y controlado, y hacia dentro, motivando a los trabajadores para que desde el puesto que ocupen hagan más grande cada día su empresa. En cada caso la respuesta de comunicación ha de salir de un gabinete, de un grupo de expertos, de un comunicador con experiencia para que el mensaje que se quiera comunicar sea creíble, fiable y eficaz.

La mayoría de los expertos en gabinetes de comunicación consideran que la comunicación en las organizaciones no se ha de improvisar, ha de seguir una estrategia establecida con anterioridad y ha de aprovechar las herramientas digitales para hacer frente a la velocidad con la que hoy circula la información en las redes, incluidas las llamadas redes sociales que se están convirtiendo en un grupo de referencia para las comunicaciones de algunas empresas y entre ellas las editoriales (Martín-Serrano, 1986; 1992; Ramírez, 1995; Álvarez-Domínguez; Caballero-Hueso, 1997, Bell-Mallén, 2004).

\section{Departamentos de comunicación editorial}

Las acciones de comunicación requieren de un departamento específico que planifique y gestione la relación con los medios (prensa, radio, televisión, webs, blogs, etc.). Editor, autor y comunicador mantendrán una comunicación constante en el tiempo que se considere para conseguir los mejores resultados. Los departamentos de comunicación tienen como objetivo principal difundir la obra mediante todos los canales posibles, elaborando el material necesario para ello. Por lo tanto, realizan una doble función interna y externa de acuerdo con la planificación estratégica. Se requiere un equilibrio externo en cuanto a la distribución de material y programación de entrevistas, de forma que todos los medios estén atendidos y reciban la información al mismo tiempo para evitar susceptibilidades.

Para algunos autores existe una relación estrecha entre las relaciones públicas, la documentación y la investigación. A juicio de Ramírez (1995: p. 82), el departamento de documentación, análisis e investigación también se encarga de difundir información a todas las personas que componen la organización, no sólo haciendo posible el acceso, sino también preparando y distribuyendo informes solicitados previamente.

Toda comunicación se realiza bajo el paraguas de un plan. En este sentido Hernández-Rodríguez (2002) señala que "los objetivos de los planes de comunicación se conciben para definir los criterios básicos de comunicación (interna, externa y marketing) durante un período largo de tiempo (por ejemplo, cuatro años) y los criterios básicos con carácter anual. Es decir, que por una parte se fijará el objetivo general de comunicación y por otro el correspondiente al año en curso, como patrón básico de trabajo".
El plan estratégico comprende varias tareas generales y específicas. Las campañas de comunicación dependen de la apuesta editorial por el libro en cuestión, bien sea un tema puntual o el resultado de la convocatoria de un premio. Este terreno es complicado por los problemas añadidos, ya que se trata de una operación de gran envergadura en la que intervienen todos los departamentos de la editorial.

\section{"El departamento de comunicación se encarga de difundir información también a todas las personas que componen la organización"}

El departamento de comunicación en las editoriales ha pasado por varias etapas. A tenor de los resultados de la investigación realizada para este trabajo, se pretende buscar soluciones integrales de comunicación en aquellos departamentos que la tienen integrada, mientras que otros aspiran a conseguir una mayor integración con el departamento de marketing. En el primer caso se encuentran los departamentos de Santilla-Alfaguara que según su responsable, Rosa Junquera, han de caminar en esa dirección: "la tendencia que se aprecia es reducir la estructura de comunicación, manteniendo un director de comunicación y/o jefe de prensa, y contratando a colaboradores y gabinetes externos para lanzamientos o campañas específicas. En cuanto al peso del departamento de comunicación depende mucho del tipo de editorial. La ficción literaria (como es nuestro caso) y el ensayo requieren mayor apoyo de prensa, mientras que la ficción y los libros de auto-ayuda requieren más soporte publicitario y de marketing". 
Por su parte, en Random House Mondadori, Leticia Rodero, aspira a conseguirlo: "creo que tiene el peso que se merece aunque debería estar más integrado con el Departamento de Marketing y Nuevas Tecnologías".

Sin embargo, la situación de crisis continuada que están experimentando los medios de comunicación no ayuda a tener más capacidad en el departamento de comunicación, ya que los propios medios están reduciendo el espacio que dedican a los libros. Así, desde SantillanaAlfaguara se apunta que "la crisis de los medios de comunicación y el momento de transición entre la prensa tradicional y la digital afecta a los departamentos de prensa. En los últimos años se ha reducido considerablemente el espacio dedicado a los libros en la prensa en general y los departamentos de comunicación tienen que adaptarse a los nuevos tiempos. Trabajamos con los medios "tradicionales" y hemos abierto intensamente el foco a la prensa digital, foros y blogs. Sin embargo, "mover" un libro en la Red supone una dedicación casi exclusiva de la que no siempre podemos disponer". De estas palabras se desprende un nuevo camino para los departamentos de comunicación, ya que al perder peso en los medios tradicionales, se les abren las puertas en los medios digitales, incluidos foros, blogs y redes sociales donde se han de hacer presentaciones y conferencias en torno a los libros que salen al mercado.

La presencia de las editoriales en Facebook es ya un hecho, sobre todo de las grandes, donde la interacción es uno de los objetivos, como indicó La Esfera de los Libros en la nota de prensa facilitada a los medios. Por otra parte, las experiencias de difusión en las redes son ya habituales, con Minotauro en la punta de lanza en junio de 2009 al emitir las primeras entregas de la novela El libro de Angelina de Fernando Figueroa-Saavedra en Facebook. Estas actividades han propiciado que en las grandes editoriales aparezca un nuevo perfil profesional que María-José Acuña denomina "responsable de contenidos digitales" y que se identifica en gran parte con el documentalista (Orúe, 2009b: p. 41).

\section{"La tendencia es reducir la estructura de comunicación, manteniendo un director y contratando a colaboradores y gabinetes externos para lanzamientos o campañas específicas"}

Frente a esta nueva realidad, las editoriales desde su departamento de comunicación consideran que su finalidad principal es crear para cada producto-libro una comunicación independiente, porque cada libro, cada autor, cada tema, cada situación por la que atraviesa el mercado supone una comunicación diferente. En el caso de Santillana-Alfaguara creen que el objetivo más inmediato es "la elaboración de planes de comunicación diseñados para cada título en función de sus características. Tratar de elaborar un 'traje a medida'. No todos los libros se comunican de la misma manera ni van orientados a los mismos medios". Por su parte, en Random House Mondadori se apuesta por las actuaciones clásicas, al valorar como importante: "conseguir gratuitamente apariciones de nuestros libros en los medios de comunicación, si bien algunos medios consideran que ciertos contenidos $\mathrm{y}$ algunos autores no son fáciles para considerar interesante esa propuesta de publicación".

Teoría y práctica no van siempre de la mano porque los tiempos son cortos y las actividades muchas, en demasiadas ocasiones. Las editoriales medianas y también algunas grandes cuentan generalmente con el departamento de comunicación en su estructura, mientras que las pequeñas contratan a empresas de servicios que se ocupen de todo el proceso. El tamaño de las empresas, determinado por la facturación, permitirá la creación y mantenimiento del departamento (tabla 1). En todos los casos el modelo de actividad es el mismo, si bien cambian las aplicaciones de acuerdo con los contenidos, los autores y las pretensiones finales.

\section{Actividad comunicativa en las editoriales}

Empieza en el momento de la creación del libro y finaliza con la muerte del mismo. Ello significa que si se mantiene vivo (reimpresiones, nuevas ediciones, relanzamientos, etc.) el departamento encargado de la comunicación tendrá siempre abierta una línea de trabajo. Por otra parte esta actividad no se refiere sólo al producto, sino que se amplía a muchos otros campos, entre los que destaca la información sobre la entidad, su forma de

\begin{tabular}{|l|c|}
\hline \multicolumn{2}{|c|}{ Tamaño de las editoriales por facturación } \\
\hline Pequeñas & $<500.000 €$ \\
\hline Pequeñas-medias & $500.000-3.000 .000 €$ \\
\hline Medias & $3.000 .000-6.000 .000 €$ \\
\hline Medias-grandes & $6.000 .000-18.000 .000 €$ \\
\hline Grandes & $>18.000 .000 €$ \\
\hline
\end{tabular}

Tabla 1 
trabajo y, por supuesto, su imagen (Martín-Armario, 1993).

\section{"En algunos centros el responsable es el jefe de prensa, en otras el director de marketing, en muchas el propio editor e incluso los comerciales"}

Los estudios de la incorporación de las nuevas tecnologías a las estrategias de comunicación realizados por Dosdoce indican la sensibilidad de las editoriales por las nuevas herramientas de la web 2.0, con datos relevantes: en 2005 un $12 \%$ de los sitios web comunicaba directamente con sus lectores, y en 2007, el 44\% de 50 editoriales analizadas ya había creado algún canal de comunicación participativa (Celaya, 2005; 2007).

http://www.dosdoce.com

La comunicación en las editoriales es realizada por profesionales cuyas tareas van más allá de las que les corresponden como periodistas o comunicadores. Ello se debe a que la globalización ha ido fusionando esas tareas, por lo que en algunos centros el responsable es el jefe de prensa, en otras el director de marketing, en muchas el propio editor e incluso los comerciales (Orúe, 2009a), situación que se produce porque en el objetivo final de la comercialización intervienen prácticamente todos los departamentos de la empresa. Incluso algunos autores tratan la comunicación desde un único punto de vista, atribuyendo tareas publicitarias (marketing) a los periodistas o comunicadores: "el departamento de comunicación actúa de intermediario entre la empresa y el mercado, en un flujo comunicativo en el que la empresa emite información sobre sus pro- ductos (comunicación publicitaria) y obtiene datos sobre sus características y las de los consumidores" (Sastre-Peláez, 2006).

Las tareas generales pasan por un primer análisis de la situación, el diseño de estrategias, los planes y programas de acción, el conocimiento de los medios, la actualización de la base de datos y el cuidado en la relación con autor y editor. Las actividades específicas serán: estudio de la obra, diseño del tipo de promoción para cada caso, gestión de la presentación del libro, gestión de los recursos para la realización de las tareas, elaboración del dossier de lanzamiento, y el seguimiento del libro una vez presentado. Todas estas actuaciones cuentan con instrumentos de apoyo: material de autopromoción, notas, reseñas, fotografías y dossieres: el modelo por excelencia que contiene las principales características para la difusión del autor y su obra.

Siguiendo los modelos de Santillana y Random House Mondadori, las tareas son coincidentes: gestión de contenidos en la web, elaboración de dossieres, presentaciones, reseñas, viajes de autores y editores, agendas de prensa (entrevistas) con los autores para dar a conocer sus libros, así como eventos y actividades en ferias del libro y la organización de tertulias literarias.

\section{"La Red ha llevado a los departamentos de prensa rapidez y eficacia"}

\section{Nuevos modelos, nuevas conductas}

Los cambios en el sector modifican los modelos y en consecuencia las conductas. Se habla, se escribe y se debate constantemente sobre los cambios en el sector editorial, pero la realidad es que las formas de actuar no han variado tanto. Los esquemas se repiten, con el apoyo tecnológico necesario, pero la sensación de los profesionales es que las conductas se mantienen. Así, la idea de contactar con el mayor número de medios de comunicación y el envío de originales a los críticos sigue siendo uno de los principales objetivos, porque las reseñas en la prensa dan prestigio al libro entre los libreros y entre el público (Memba, 2000).

La globalización es también interna y las líneas diferenciales entre secciones tienden a concentrarse, de forma que las tareas de marketing y comunicación se funden en lo que el modelo americano llama marcom. En la editorial Espasa, por ejemplo, el departamento se denomina Marketing y Comunicación, con un responsable común, aunque en la práctica los trabajos se diversifican y se encargan a especialistas en cada materia, tanto internos como externos.

Actualmente los equipos realizan todo tipo de trabajos: promoción, reseñas, presentación a los medios, dossieres, folletos, folders, investigación en la Red, actos culturales, etc., trabajo tan intenso que requiere personal dinámico y creativo, capaz de soportar la presión y al mismo tiempo continuar con su ritmo.

La cuestión clave es si realmente se han modificado los sistemas de trabajo en los departamentos de comunicación y, de haberse producido tal cambio, en qué medida ha influido internet. Las actividades tradicionales ceden espacio y tiempo a las nuevas tareas. El teléfono pierde protagonismo y los dossieres se redactan para su inclusión en la web de la editorial. Ahora se dedica gran parte del tiempo a las actualizaciones de contenidos de la web, a la grabación de vídeos o sesiones fotográficas con los autores, aten- 
der los blogs o consultar los medios para recoger las repercusiones. En este sentido, todos los profesionales se muestran de acuerdo: la Red ha llevado a los departamentos de prensa rapidez y eficacia. Este es el presente y el futuro, porque las posibilidades se abren a portales, webs de autores, blogs literarios o a las redes sociales. Las iniciativas son muchas, pero todavía con escasos resultados.

La difusión en estos sistemas permite un intercambio que genera interactividad; sin embargo todavía no se ha estudiado la repuesta de los usuarios. De esta nueva situación surgen dos preguntas: ¿el usuario da credibilidad a lo que se presenta o lo considera estrategia publicitaria?, ¿es fiable la información que se ofrece desde la editorial? Este es uno de los puntos cruciales, la credibilidad, próxima a la ética profesional.

Otra cuestión de interés es la valoración que los profesionales hacen de la comunicación en los medios impresos frente a internet. Si lo que se pretende es difundir el libro en el mayor radio posible, la competencia con la Red es imposible, y en este sentido las preferencias están claras. Es obvio que la mayoría de los autores prefieren que su obra se reseñe en un blog o foro con miles de visitas antes que en un suplemento cultural reservado a la elite cultural.

Por último cabe señalar que se acentúa la externalización de las tareas comunicativas, es decir el encargo a empresas de servicios. Este aspecto es fundamental para entender determinadas actuaciones, ya que dependen de los objetivos generales de la editorial. Ello supone la absoluta confianza en el profesional y la fidelidad de éste; es decir, que se hace necesaria una complicidad total. Lola Martín, directora de Literal Press y responsable de comunicación de Trea, indica que el proceso es el mismo dentro y fuera, y que las actuaciones se diferencian por los contenidos y no por otros motivos.

\section{"De la aplicación y el uso de los nuevos medios ha surgido una nueva figura profesional: el gestor de contenidos digitales"}

\section{Conclusiones}

- Las acciones de comunicación han modificado los modelos gracias a internet, que ha propiciado el cambio en la comunicación y el marketing en las empresas editoriales.

- Las redes sociales se presentan como el medio fundamental en la comunicación de los productos editoriales.

- Los sistemas de trabajo son similares en las actuaciones básicas pero diferentes en las aplicaciones prácticas según los contenidos.

- La interactividad con los usuarios es una de las pretensiones fundamentales de los responsables de la comunicación.

- De la aplicación y el uso de los nuevos medios ha surgido una nueva figura profesional: el gestor de contenidos digitales.

\section{Bibliografía}

Álvarez-Domínguez, Tomás; Caballero-Hueso, Mercedes. Vendedores de imagen: los retos de los nuevos gabinetes de comunicación. Barcelona: Paidós, 1997.

Bel-Mallén, José-Ignacio. Comunicar para crear valor: la dirección de comunicación en las organizaciones. Navarra: Eunsa, 2004.

Camacho-Bellón, José-Antonio. "El marketing en el sector editorial". Delibros, 2003, junio, n. 166, pp. 30-32.

Celaya, Javier. La comunicación en la promoción del libro, 2005.

http://www.dosdoce.com

Celaya, Javier. Las nuevas tecnologías web 2.0, 2006.

http://www.dosdoce.com
Celaya, Javier. Tendencias web 2.0 en el sector editorial, 2007.

http://www.dosdoce.com/continguts/estudios/ vistaSola_cas.php? ID=18

Cole, David. Marketing editorial. México: Fondo de Cultura Económica, 2003.

Hernández-Rodríguez, Ana J. "Planificar la comunicación". Revista latina de comunicación social, 2002, n. 48.

http://www.ull.es/publicaciones/latina/2002/ latina48marzo/4812ajhernandez.htm

Hoyuela-Sánchez, Paloma. La comunicación en la empresa editorial. Tesis dirigida por Rafael López-Lita. Facultad de Ciencias de la Información de la UCM. Departamento de Comunicación Audiovisual y Publicidad 1, 1997.

http://eprints.ucm.es/tesis/19972000/S/3/ S3031101.pdf

Marcos-Recio, Juan-Carlos; García-Jiménez, Antonio; Nuño-Moral, María-Victoria. Gestión de la documentación en la publicidad y en las relaciones públicas. Madrid: Síntesis, 2004.

Martín-Armario, Enrique. Marketing. Barcelona: Editorial Arte, 1993, pp. 453-457.

Martín-Serrano, Manuel. "Los cambios acontecidos en las funciones de la comunicación y en el valor de la información", Reis, 1992, eneromarzo, n. 57.

http://www.reis.cis.es/REIS/PDF/REIS_057_ 04.pdf

Martín-Serrano, Manuel. La producción social de comunicación. Madrid: Alianza Editorial, 1986.

Memba, Javier. Cómo se hacen los libros (VI). La promoción, el arma clave, 2000, julio.

http://www.elmundo.es/elmundolibro/2000/07/09/ anticuario/962962416.html

Orúe, Eva. "La publicidad editorial en internet". Delibros, marzo, 2009a, n. 229, pp. 20-25.

Orúe, Eva. "La promoción editorial salta a la Red". Delibros, octubre, 2009b, n. 235, pp. 4051 .

Ramírez, Txema. Gabinetes de comunicación. Funciones, disfunciones e incidencias. Barcelona: Bosch, 2005.

Sánchez-Vigil, Juan-Miguel. La edición en España. Industria cultural por excelencia: historia, proceso, gestión, documentación. Gijón: Trea, 2009.

Sastre-Peláez, Francisco-Luis. La empresa en su resultado: el beneficio y la contabilidad del conocimiento. Tesis doctoral. Facultad Ciencias Económicas y Empresariales de la Universidad Pontificia de Comillas, 2006.

http://www.eumed.net/tesis/2006/flsp

Juan-Carlos Marcos-Recio, JuanMiguel Sánchez-Vigil, María Olivera-Zaldua. Universidad Complutense de Madrid. jmarcos@ccinf.ucm.es jmvigil@ccinf.ucm.es molizal@hotmail.com 